\title{
Detection of R Peak in Electrocardiogram
}

\author{
Pankhuri Trivedi \\ M. Tech Scholar \\ Department of Electronics \& Communication \\ Engineering \\ B.I.E.T, Jhansi (U.P)
}

\author{
Shahanaz Ayub, PhD \\ Associate Professor and Head \\ Department of Electronics \& Communication \\ Engineering \\ B.I.E.T, Jhansi (U.P)
}

\begin{abstract}
Electrocardiogram (ECG) is the recording of electrical activity of the heart. Accurate measurement of ECG parameters is an important requirement of ECG analysis, particularly if the results are to be used for clinical purposes. Most automatic ECG diagnosis techniques require an accurate detection of the QRS complex. Almost all the analysis methods use the standard Massachusetts Institute of Technology-Beth Israel Hospital (MIT-BIH) database which is available in the digital form. In this paper a method for detection of R peak of ECG signal is discussed. The main objective of this work is to test this method using ECG of Indian patients. ECG signals from Modified Lead II (MLII) are chosen for processing.
\end{abstract}

\section{Keywords}

ECG, Beat Detection, P-QRS-T waves, Windows, MATLAB.

\section{INTRODUCTION}

Electrocardiography (ECG) is a tool that is widely used to understand the condition of the heart, since it records the electrical activity generated over the cardiac cycle via electrodes positioned at various locations on the body surface. Fig.1 shows an example of a normal ECG signal, which consists of a $\mathrm{P}$ wave, a QRS complex and a $\mathrm{T}$ wave. The small $\mathrm{U}$ wave may also be sometimes visible. The $\mathrm{P}$ wave corresponds to the current which leads to atrial contraction, the QRS complex represents the current that causes contraction of the left and right ventricles, the $\mathrm{T}$ wave depicts the repolarization of the ventricles, and the $U$ wave is considered to be a representation of the papillary muscles or Purkinje fibers that corresponds to the ventricular contraction. The automatic detection of QRS Complex in an ECG signal is the first crucial step in the automatic description of ECG signal. Accurate determination of the QRS complex, in particular, accurate detection of the $\mathrm{R}$ peak, is essential in computer-based ECG analysis. For processing ECG signal the first step is to implement heartbeat detection. Heartbeat refers to a single complete pulsation of the heart during when cardiac muscles contract and subsequently relax. In the ECG one pulsation is referred to as the characteristics of ECG waveform between two successive $\mathrm{R}$ peaks. The detection of $\mathrm{R}$ peak is associated with detection of $\mathrm{QRS}$ complex that ultimately detects QRS complex. For evaluation of ECG parameters, internationally-recognized databases with ECG signals and reference annotations have been used. Most of them are listed on PhysioNet .The following three databases served for QRS detection:
1. MIT DB: The Massachusetts Institute of Technology- Beth Israel Hospital Arrhythmia Database

2. AHA DB: The American Heart Association ECG Database

3. ESC DB: The European Society of Cardiology ST-T

Database

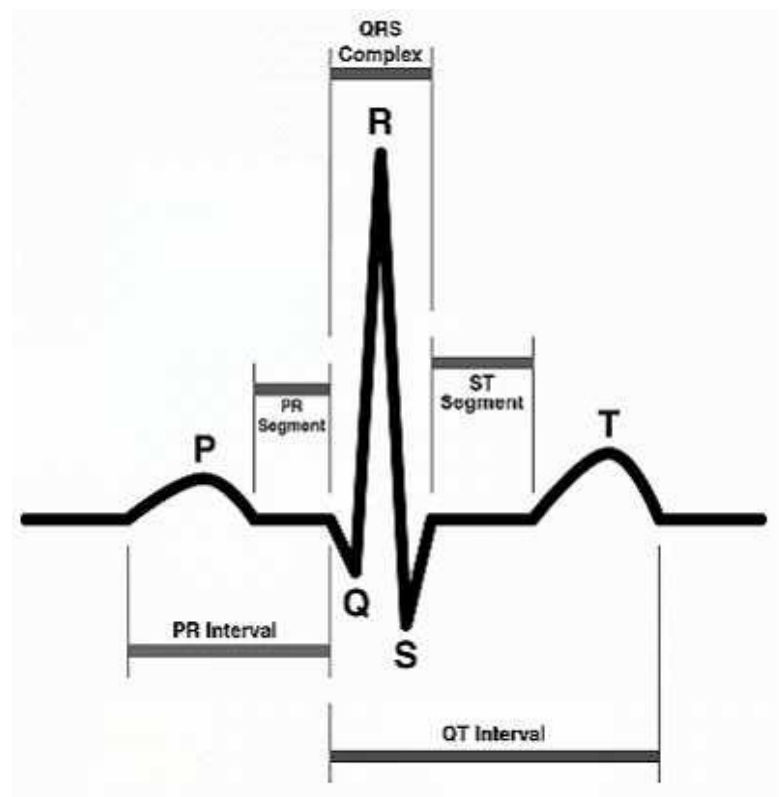

Fig. 1: The ECG signal

QRS detection is difficult, not only because of the physiological variability of the QRS complexes, but also because of the various types of noise that can be present in the ECG signal. Noise contamination, due to baseline drifts changes, motion artifacts and muscular noise, is frequently encountered [1]. A real time algorithm for detection of the QRS complex is developed in which threshold adjusts automatically to adapt ECG changes as QRS morphology and heart rate [2]. The slope of the $\mathrm{R}$ wave is a popular signal feature used to locate the QRS complex [3]. For the purpose of ECG analysis, a reliable QRS detection algorithm based on the maximum slope detection was developed [4]. Several modifications have been done on Pan Tompkins' algorithm in subsequent years. In order to obtain the best extraction of the QRS complex, baseline correction is required. So, a fixed thresholding is required instead of adaptive thresholding [5]. A modified Pan Tompkins algorithm by using a SavitzkyGolay filter in place of the high pass filter and differentiator of Pan Tompkins' algorithm is developed [6]. All the parameters 
of ECG are measured by using Pan Tompkins algorithm by implementing it in MATLAB [7]. Digital filters for the removal of baseline wandering from ECG signal is designed using FIR filters with window techniques [8].

In this work ECGs of Indian patients are taken using BPL 108T machine and they are converted to digital form with the help of MATLAB software. The ECG signals are digitized with sampling frequency of $295 \mathrm{~Hz}$. ECG signals from Modified Lead II (MLII) are chosen for processing. In this work a slight modification is done on Pan Tompkins algorithm to determine the location of R peak of ECG signal.

\section{ALGORITHM OVERVIEW}

A Simulink model has been developed for the detection of $\mathrm{R}$ peak. First, in order to attenuate noise, the signal passes through a digital FIR band pass filter. The next process after filtering is differentiation, followed by squaring and then windowed integration. Information about the slope of the QRS is obtained in the derivative stage. The squaring process intensifies the slope of the frequency response of the derivative. The filter that is used is a digital FIR band pass filter with sampling frequency $295 \mathrm{~Hz}$, lower and upper cut off frequencies of $15 \mathrm{~Hz}$ and $17 \mathrm{~Hz}$ respectively, the windowing technique employed is Kaiser Window. The simulink model for the proposed algorithm is as follows:

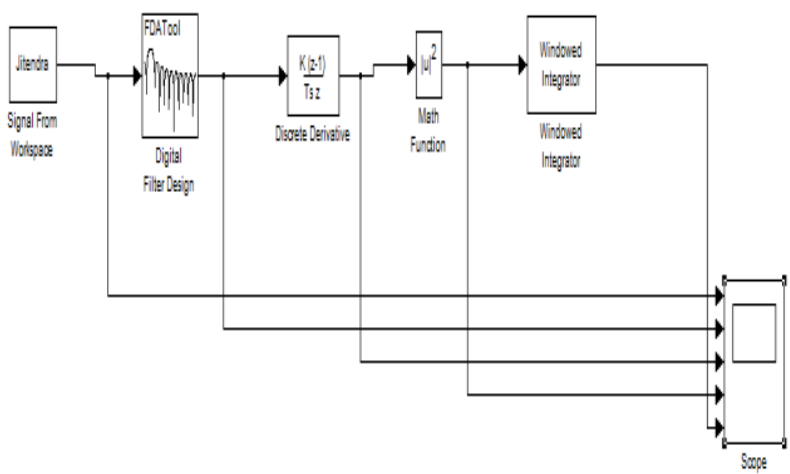

Fig. 2: Simulink model of the proposed algorithm

The magnitude response of the filter is given in Fig. 3 .

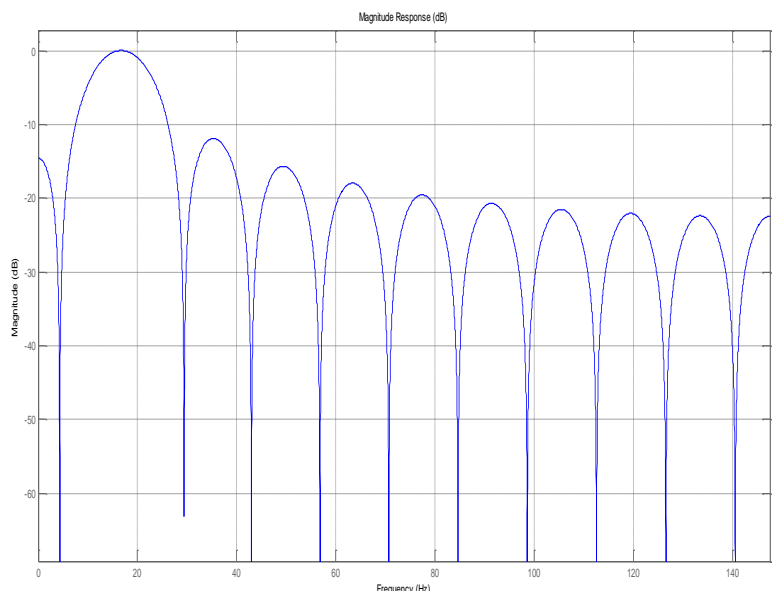

Fig. 3: Magnitude response of the filter

Kaiser Window has been used in this case for the digital FIR band pass filter with $\beta=0.5$. The time domain and frequency domain response is given in Fig 4.
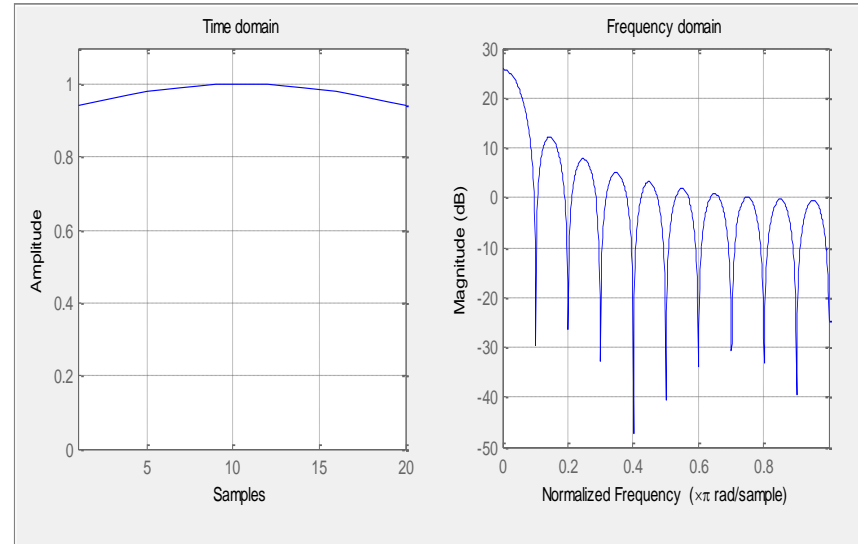

Fig. 4: Time $\&$ frequency domain responses of Kaiser Window

For detection of the $\mathrm{R}$ peak the output of the windowed integrator is used. The output of windowed integrator is firstly passed through a low pass filter for removal of noise and then using MATLAB code, the R peak is detected where the sign of the slope is changing for this output.

\section{RESULTS}

Fig 5 shows the original ECG signal before processing.

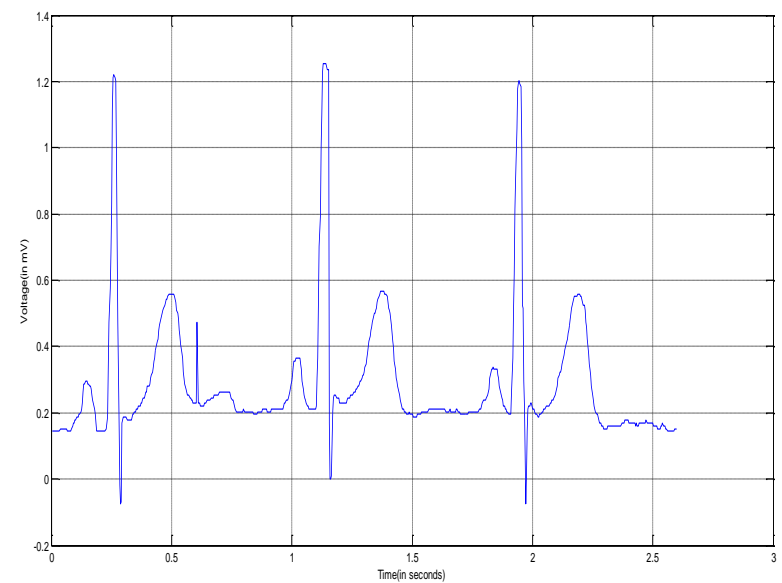

Fig.5: ECG Signal

Next, the ECG signal is passed through a FIR band pass filter using Kaiser Window, whose result is shown in Fig 6. By performing this particular operation, some portion of the noise in the ECG signal is eliminated and we get the QRS complex which is the main useful part of the ECG wave. 


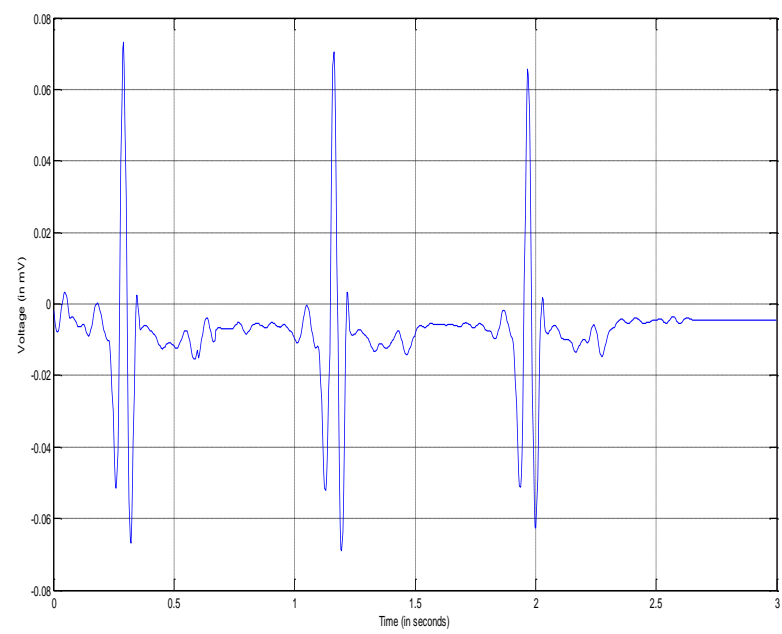

Fig 6: ECG Signal after Filtering

After this, the filtered ECG signal is passed through a differentiator. The signal is differentiated to obtain the slope information about the QRS complex. Fig 7 shows the differentiated output.

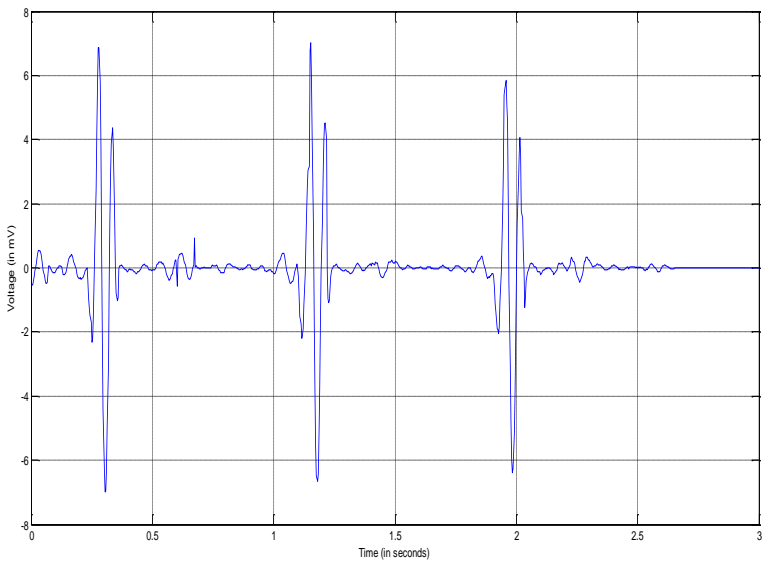

Fig 7: Differentiator output

Next, the differentiated signal is squared. By squaring, all the data points become positive and it does non-linear amplification of the output of the derivative by considering the higher frequencies. Fig 8 shows the squared output.

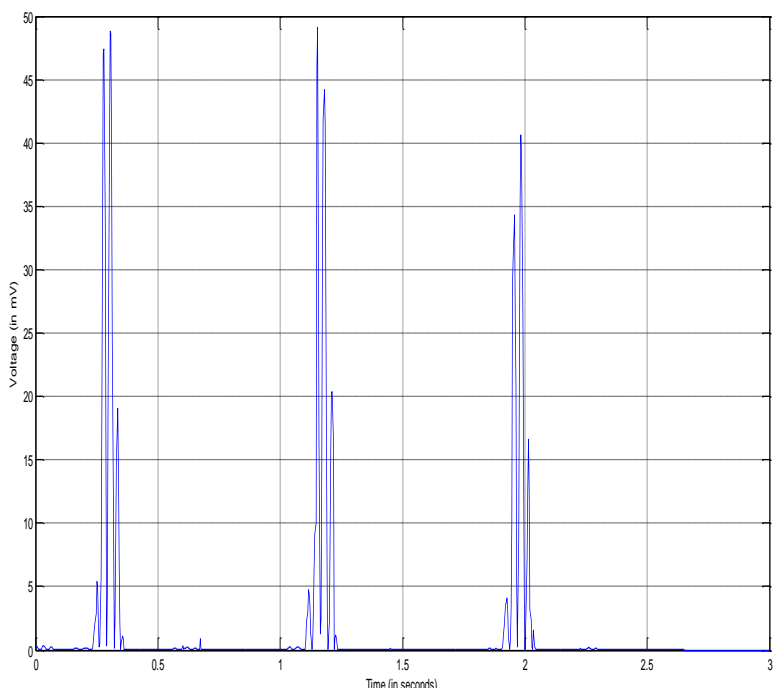

Fig 8: Squaring output
Next, the signal is passed through a windowed integrator. From this, we can obtain the slope of the R wave. Fig 9 shows the moving window integrator output.

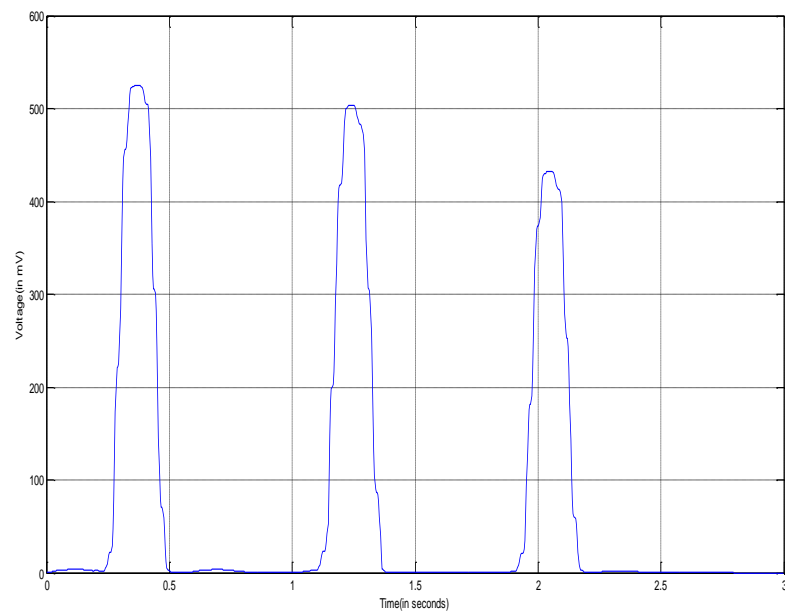

Fig 9: Moving window integrator output

Here, the number of samples of the windowed integrator is taken as 44. It is calculated by using an empirical formula that for a sample rate of 200 samples/s the number of samples taken is 30 .

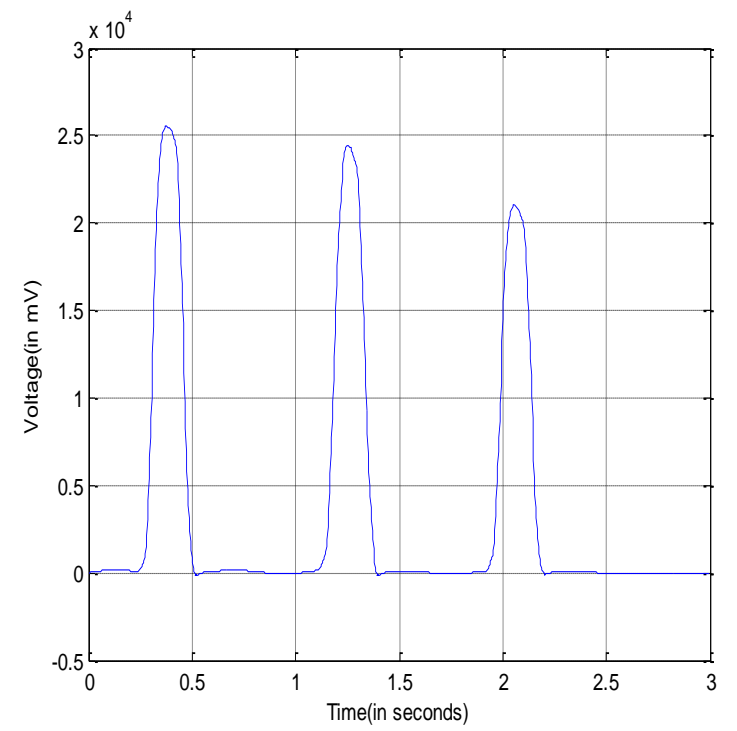

Fig 10: Output of moving window integrator after filtering

The output of the windowed integrator does not suffice for the total work. It contains noise and so the peak detection can be difficult. For this purpose, the output of windowed integrator is filtered so that we get a smooth curve, which is shown in Fig 10. Now with the help of MATLAB, the time instants for the corresponding $\mathrm{R}$ peaks are detected when the sign of the slope is changing.

The following tables give the actual and measured location of $R$ peak and the relative error in calculating the RR interval. 


\begin{tabular}{|c|c|c|}
\hline R peak & $\begin{array}{c}\text { Measured Time } \\
(\mathbf{s e c})\end{array}$ & $\begin{array}{c}\text { Actual Time } \\
(\mathbf{s e c})\end{array}$ \\
\hline 1st peak & 0.3752 & 0.3402 \\
\hline 2nd peak & 1.254 & 1.213 \\
\hline 3rd peak & 2.055 & 2.022 \\
\hline
\end{tabular}

Table 1: Time instants of $R$ peak

\begin{tabular}{|c|c|c|c|}
\hline $\begin{array}{c}\text { RR } \\
\text { interval }\end{array}$ & $\begin{array}{c}\text { Actual } \\
\text { Value }\end{array}$ & $\begin{array}{c}\text { Measured } \\
\text { Value }\end{array}$ & $\begin{array}{c}\text { Relative } \\
\text { Error }\end{array}$ \\
\hline RR1 & $870.5 \mathrm{~ms}$ & $878.8 \mathrm{~ms}$ & $0.9 \%$ \\
\hline RR2 & $812.8 \mathrm{~ms}$ & $801 \mathrm{~ms}$ & $1.4 \%$ \\
\hline
\end{tabular}

Table 2: RR Interval (actual and measured) and the relative error

\section{CONCLUSION}

The location of the $\mathrm{R}$ peak is detected using proposed algorithm. The error in the location of the $\mathrm{R}$ peaks is within the permissible limit. So the algorithm is acceptable for ECG signal processing. Using the location of the $\mathrm{R}$ peaks the $\mathrm{RR}$ interval can also be calculated and on the basis of the measured value cardiac arrhythmias can be detected. The algorithm can be modified further to calculate all the ECG parameters automatically at the output. Thus, it can be used for the processing of real time ECG signals in future.

\section{REFERENCES}

[1] G.M. Friesen, T.C. Jannett, M.A. Jadallah, S.L. Yates, S.R. Quint, H.T. Nagle, "A comparison of the noise sensitivity of nine QRS detection algorithms", IEEE Trans. Biomed. Eng. 37 (1990) 85-98.

[2] Pan J and Tompkins W J, 1985, "A Real Time QRS Detection Algorithm” IEEE Trans BME- 32,230-236

[3] M.L. Ahlstrom and W.J. Tompkins, "Automated highspeed analysis of Holter tapes with microcomputers," IEEE Trans. Biomed. Eng., vol. BME-30, pp.651657,Oct. 1983.

[4] So H. H. and Chan K. L., 1997, "Development Of QRS Detection Method for Real Time Ambulatory Cardiac Monitor", $19^{\text {th }}$ Annu Int Conf IEEE/EMBS, Chicago, USA, 289-292.

[5] Nehla Debbabi, Sadol ElAsmi, Hichem Arfa,2010,"Correction of ECG baseline wander Application to the Pan \& Tompkins QRS detection algorithm", $5^{\text {th }}$ international symposium on Communication and mobile network, DOI: 10.1109/ISVC.2010.5654714, pp. 1-4.

[6] Das, Shreya and Chakraborty, Monisha , "QRS Detection Algorithm Using Savitzky-Golay Filter", ACEEE Int J on Signal and Image Processing, Vol. 03,No. 01,Jan 2012.

[7] Singh Sachin and Gandhi N. Netaji, 2010, "Pattern Analysis of Different ECG Signal Using Pan Tompkin's algorithm", International Journal on Computer Science and Engineering. Vol. 02, No. 07, pp. 2502-2505.

[8] Lakhwani, R.; Ayub, S; Saini, J.P. "Design and Comparison of Digital Filters for Removal of Baseline Wandering from ECG Signal" Computational Intelligence and Communication Networks (CICN), IEEE 2013 Digital Object Identifier: 10.1109/CICN.2013. 48 page(s): 186-191. 